\title{
Testing SUSY models for the muon g-2 anomaly via Chargino-Neutralino Pair Production at the LHC
}

\author{
Siba Prasad Das ${ }^{a}$, Monoranjan Guchait ${ }^{b}$ and D. P. Roy ${ }^{c}$ \\ a) Institute of Physics, Sachivalaya Marg, \\ Bhubaneswar 751 005, Orissa, India. \\ b) Department of High Energy Physics, Tata Institute of Fundamental Research, \\ 1, Homi Bhabha Road, Mumbai 400 005, India. \\ c) Homi Bhabha's Centre for Science Education, Tata Institute of Fundamental Research \\ V. N. Purav Marg, Mumbai-400088, India.
}

\begin{abstract}
Non-universal gaugino mass models can naturally account for the dark matter relic density via the bulk annihilation process with relatively light bino LSP and right sleptons in the mass range of $\sim 100 \mathrm{GeV}$, while accommodating the observed Higgs boson mass of $\sim 125 \mathrm{GeV}$ with $\mathrm{TeV}$ scale squark/gluino masses. A class of these models can also account for the observed muon g-2 anomaly via SUSY loops with wino and left sleptons in the mass range of $400-700 \mathrm{GeV}$. These models can be tested at LHC via electroweak production of charged and neutral wino pair, leading to robust trilepton and same sign dilepton signals. We investigate these signals along with the standard model background for both 8 and $13 \mathrm{TeV}$ LHC runs.
\end{abstract}




\section{Introduction}

A large part of the SUSY phenomenology over the past years has been based on the minimal supergravity or the so-called constrained minimal supersymmetric standard model (CMSSM), which assumes universal gaugino and scalar masses $m_{1 / 2}$ and $m_{0}$ at the GUT scale [1-3] . In this case the lightest superparticle (LSP), i.e., the dark matter, is dominantly a bino over most of the model parameter space. Since the bino does not carry any gauge charge, its main annihilation process is via sfermion exchange into a pair of fermions. And the cosmologically compatible dark matter relic density requires rather small bino and sfermion masses $\sim 100 \mathrm{GeV}$. This is the so-called bulk annihilation region. Unfortunately, the LEP limit on the light Higgs boson mass, $m_{h}>114$ $\mathrm{GeV}$ practically rules out the bulk annihilation region of the CMSSM as it requires TeV scale squark/gluino masses [4]. This is further reinforced now with the reported discovery of Higgs boson at LHC by the ATLAS and CMS experiments $[5,6]$ at

$$
m_{h} \simeq 125 \mathrm{GeV} \text {. }
$$

It was shown in [7] that the natural explanation of the cosmologically compatible dark matter relic density in the bulk annihilation region can be reconciled with the Higgs boson mass limit from LEP in a class of simple and well motivated MSSM with non-universal gaugino masses(NUGM) based on $\mathrm{SU}(5) \mathrm{GUT}[8,9]$. In these models one can have relatively small bino and right slepton masses in the range of $\sim 100 \mathrm{GeV}$ to account for the former along with $\mathrm{TeV}$ scale squark/gluino masses to account for the latter. Moreover, these models can raise the Higgs boson mass to the observed range of $\sim 125 \mathrm{GeV}$ with the help of a TeV scale tri-linear coupling term $A_{0}$ [10]. More recently it was shown in [11] that some of these models have relatively modest wino and left slepton masses in the range of 400-700 GeV, so that they can also account for the reported muon g-2 anomaly [12,13] via wino-left slepton loops [14-18].

In this work we investigate the prospect of probing the above mentioned mass range of 400$700 \mathrm{GeV}$ for wino and left sleptons in these models at the $8 \mathrm{TeV}$ and the forthcoming $13 \mathrm{TeV}$ runs of the LHC. Section 2 gives a brief description of the non-universal gaugino mass models based on SU(5) GUT. Section 3 discusses the weak scale SUSY spectra and muon g-2 prediction for two such models, where the wino and left slepton lie in the mass range of $400-700 \mathrm{GeV}$. In particular we shall list them for a few benchmark points of these models for computing the LHC signal. Section 4 describes the electroweak production of charged and neutral wino pair, leading to distinctive trilepton and same sign di-lepton signals at the LHC. It also discusses the selection cuts used in this analysis to extract these signals from the main standard model background. Section 5 discusses the results of our analysis of these two channels for both the $8 \mathrm{TeV}$ and the forthcoming $13 \mathrm{TeV}$ runs of LHC. We conclude with a summary in section 6.

\section{Non-universal Gaugino Mass model in SU(5) GUT}

The gauge kinetic function responsible for the gaugino masses in the GUT scale Lagrangian originates from the vacuum expectation values of the F-term of a chiral superfield $\Omega$ responsible for SUSY breaking,

$$
\frac{<\mathrm{F}_{\Omega}>}{\mathrm{M}_{\text {planck }}} \lambda_{\mathrm{i}} \lambda_{\mathrm{j}}
$$


where $\lambda_{1,2,3}$ are the $\mathrm{U}(1), \mathrm{SU}(2)$ and $\mathrm{SU}(3)$ gaugino fields- bino, wino and gluino respectively. Since gauginos belong to the adjoint representation of the GUT group, $\Omega$ and $F_{\Omega}$ can belong to any of the irreducible representations occurring in their symmetric product, i.e.,

$$
(24 \times 24)_{\text {sym }}=1+24+75+200
$$

for the simplest GUT group SU(5). Thus for a given representation of the SUSY breaking superfield, the GUT scale gaugino masses are given in terms of one mass parameter as $[8,9]$

$$
\mathrm{M}_{1,2,3}^{\mathrm{G}}=\mathrm{C}_{1,2,3}^{\mathrm{n}} \mathrm{m}_{1 / 2}^{\mathrm{n}}
$$

where

$$
C_{1,2,3}^{1}=(1,1,1), C_{1,2,3}^{24}=(-1,-3,2), C_{1,2,3}^{75}=(-5,3,1), C_{1,2,3}^{200}=(10,2,1) .
$$

The CMSSM assumes $\Omega$ to be a singlet, leading to universal gaugino masses at the GUT scale. On the other hand, any of the non-singlet representations of $\Omega$ would imply non-universal gaugino masses at the GUT scale via eqs. 4 and 5. These non-universal gaugino masses are known to be consistent with the universality of gauge couplings at the GUT scale $[8,9,19]$ with $\alpha_{G} \simeq \frac{1}{25}$. The phenomenology of non-universal gauginos arising from each of these non-singlet $\Omega$ have been widely studied [20-22].

It was assumed in [7] that SUSY is broken by a combination of a singlet and a non-singlet superfields belonging to the $1+24,1+75$ or $1+200$ representations of SU(5). Then the GUT scale gaugino masses are given in terms of two mass parameters,

$$
\mathrm{M}_{1,2,3}^{\mathrm{G}}=\mathrm{C}_{1,2,3}^{1} \mathrm{~m}_{1 / 2}^{1}+\mathrm{C}_{1,2,3}^{\ell} \mathrm{m}_{1 / 2}^{\ell}, \quad \ell=24,75,200 .
$$

which are determined by the two independent VEVs of the F terms of the singlet and the nonsinglet superfields. The corresponding weak scale superparticle and Higgs boson masses are fixed in terms of these two gaugino mass parameters and the universal scalar mass parameter $m_{0}$ along with the tri-linear coupling $A_{0}$ via the RGE. In these models one could access the bulk annihilation region of dark matter relic density, while keeping the Higgs boson mass above the LEP limit of $114 \mathrm{GeV}$ [7] and raise it further to the LHC value of $\sim 125 \mathrm{GeV}$ with the help of a TeV scale $A_{0}$ parameter [10]. To understand this result, one can equivalently consider the two independent gaugino mass parameter of eq.6 in any of these three models to be $M_{1}^{G}$ and $M_{3}^{G}$. The corresponding weak scale gaugino masses are given to a good approximation by the one loop RGE,

$$
\mathrm{M}_{1,2,3}=\frac{\alpha_{1,2,3}}{\alpha_{\mathrm{G}}} \mathrm{M}_{1,2,3}^{\mathrm{G}} \simeq \frac{25}{60,30,9} \mathrm{M}_{1,2,3}^{\mathrm{G}} .
$$

Thus one can choose a relatively small $M_{1}^{G} \sim 200 \mathrm{GeV}$ along with a small $m_{0} \sim 100 \mathrm{GeV}$ to ensure a small weak scale bino mass $M_{1} \sim 80 \mathrm{GeV}$ along with right slepton masses $\sim 100 \mathrm{GeV}$. Then the annihilation of the bino LSP pair via right slepton exchange

$$
\chi \chi \stackrel{\tilde{\ell}_{R}}{\rightarrow} \bar{\ell} \ell
$$

gives the desired dark matter relic density. The other mass parameter $M_{3}^{G}$ can then be raised to an appropriate level to give $\mathrm{TeV}$ scale squark/gluino masses as required by the Higgs boson mass of $\sim 125 \mathrm{GeV}$ and the negative squark/gluino search results from LHC.

The issue of naturalness for these nonuniversal gaugino mass models has been discussed in $[7,23]$ via the fine-tuning parameters, $\Delta^{\Omega}$ and $\Delta^{\mathrm{EW}}$, required for achieving the right dark matter relic 
density and radiative EW symmetry breaking. It was found that $\Delta^{\Omega} \sim 1$ over the bulk annihilation region of these models, which means there is no fine-tuning required in achieving the right dark matter relic density. In contrast the allowed dark matter compatible regions of the CMSSM or the nonuniversal scalar mass models had 1-2 orders of magnitude higher values of this fine -tuning measure. Of course, one has to pay the usual fine-tuning price for radiative symmetry breaking, $\Delta^{\mathrm{EW}} \sim 10^{2}$, in the dark matter compatible regions of all these MSSM. However, a quantitative evaluation of this fine-tuning parameter in [23] showed that the bulk annihilation region of the nonuniversal gaugino mass models had one of the lowest $\Delta^{\mathrm{EW}}$ of them all. Thus the low value of $\Delta^{\Omega}$ is achieved here without any additional cost to the $\Delta^{\mathrm{EW}}$.

Note that with given $M_{1}^{G}$ and $M_{3}^{G}$ inputs, each of the above three models makes a definitive prediction for $M_{2}^{G}$. It follows from eqs. 5 and 6 that the $(1+200)$ model predicts the smallest $M_{2}^{G}$ and hence the smallest weak scale wino and left slepton masses among all the three models. Hence it offers the best chance to account for a significant SUSY contribution to the muon g-2 anomaly, as discussed in [11]. As further discussed in [11], one can extend the analysis to a general nonuniversal gaugino mass model with three independent gaugino mass parameters, $M_{1}^{G}, M_{2}^{G}$ and $M_{3}^{G}$. This can be realized in a scenario of SUSY breaking by three superfields, belonging to different representations of the GUT group, e.g., a $(1+75+200)$ model. The three gaugino mass parameters are linearly related to the VEVs of the F terms of these three superfields. In this case one can have very modest wino and left slepton masses $\sim 400 \mathrm{GeV}$, so as to give a SUSY contribution to the muon g-2 anomaly very close to its experimental central value. In the next section we shall focus on some benchmark points from these two models, which can account for the muon g-2 anomaly within $2 \sigma$ level.

\section{The weak scale SUSY spectra and muon g-2 contributions in the $(1+200)$ model and the general non-universal gaugino mass Model}

We have used the two-loop RGE code in SusPect [24] to generate the weak scale SUSY and Higgs spectra. One should note that the $\overline{\mathrm{MS}}$ renormalization scheme used in the SusPect RGE code is known to predict a lower Higgs boson mass than the on-shell renormalization scheme used in FeynHiggs [25] by $2-3 \mathrm{GeV}$ [26-30]. Therefore a predicted Higgs boson mass $\gtrsim 122 \mathrm{GeV}$ in the following tables is compatible with the reported mass of $\sim 125 \mathrm{GeV}[5,6]$.

The resulting dark matter relic density and the muon anomalous magnetic moment $(\mathrm{g}-2)$ were computed using the microOMEGAs code [31-33]. In view of the high precision of the dark matter relic density data [34] we have considered solutions lying within $3 \sigma$ of its central value as in [11], i.e.,

$$
0.102<\Omega h^{2}<0.123
$$

On the other hand the measured value [12] of the muon anomalous magnetic moment excess has a relatively large uncertainty,

$$
\Delta a_{\mu}=(28.7 \pm 8.0) \times 10^{-10}
$$

where

$$
a_{\mu}=\frac{(g-2) \mu}{2} .
$$


3 The weak scale SUSY spectra and muon g-2 contributions in the $(1+200)$ model and the general non-universal gaugino mass Model

\begin{tabular}{|c|c|c|c|c|c|c|c|c|}
\hline Parameters & $m_{0}$ & $\tan \beta$ & $A_{t 0}=A_{b 0}$ & $M_{1}^{G}$ & $M_{2}^{G}$ & $M_{3}^{G}$ & $a_{\mu}^{\text {SUSY }}$ & $\delta a_{\mu}$ \\
\hline BP1 & 103 & 15 & -2.4 & 200 & 734.3 & 800 & $1.59 \times 10^{-9}$ & $1.61 \sigma$ \\
BP2 & 103 & 15 & -2.4 & 200 & 822.2 & 900 & $1.37 \times 10^{-9}$ & $1.89 \sigma$ \\
BP3 & 138 & 15 & -2.4 & 200 & 575 & 1200 & $2.67 \times 10^{-9}$ & $0.26 \sigma$ \\
\hline
\end{tabular}

Table 1: Benchmark points of SUSY parameter space taken from Ref. [11] to simulate signal process(all masses are in $\mathrm{GeV}$ and A parameters are in $\mathrm{TeV}$ ). The corresponding SUSY contributions to muon anomalous magentic moment are shown along with their differences from the measured central value of eq. 10.

\begin{tabular}{|c|c|c|c|c|c|c|c|c|c|c|c|c|}
\hline Point & $\tilde{g}$ & $\tilde{q}_{L}$ & $\tilde{q}_{R}$ & $\tilde{t}_{1,2}$ & $\tilde{b}_{1}$ & $\tilde{\ell}_{L, R}$ & $\tilde{\tau}_{1,2}$ & $\chi_{1}^{0}$ & $\chi_{2}^{0}$ & $\chi_{1}^{+}$ & $\chi_{2}^{+}$ & $h$ \\
\hline BP1 & 1764 & 1600 & 1540 & 820,1531 & 1311 & 479,133 & 94,480 & 80 & 593 & 593 & 1494 & 124 \\
BP2 & 1967 & 1778 & 1711 & 1012,1524 & 1490 & 531,132 & 90,532 & 80 & 666 & 666 & 1584 & 124 \\
BP3 & 2578 & 2252 & 2235 & 1596,1994 & 1967 & 380,159 & 96,396 & 78 & 461 & 461 & 1871 & 123 \\
\hline
\end{tabular}

Table 2: Masses of SUSY particles(in GeV) calculated using SuSpect v2.41 [24] for the three benchmark points given in Table 1.

Therefore we have considered SUSY solutions to $a_{\mu}$ [11] lying within $2 \sigma$ of the central value, i.e.,

$$
\delta a_{\mu}=\Delta a_{\mu}-a_{\mu}^{S U S Y}<2 \sigma
$$

so that $a_{\mu}^{S U S Y}$ is at least of the same order as the central value of the experimental excess of eq. 10.

Explicit formulae for $a_{\mu}^{S U S Y}$, arising from wino-left slepton and bino-right slepton loops, can be found e.g., in $[14,15]$. It increases linearly with $\tan \beta$ at constant SUSY masses. However, one has to choose a higher $m_{0}$ at larger $\tan \beta$ to maintain the $\widetilde{\tau}_{1}$ mass in the desired range for the dark matter relic density of eq. 9. The resulting increase in slepton masses compensate the linear rise of $a_{\mu}^{S U S Y}$ with $\tan \beta$. This results in a broad peak of $a_{\mu}^{S U S Y}$ at $\tan \beta \simeq 15$, which remains nearly constant over the moderate $\tan \beta(=10-20)$ regions [11]. Therefore we have chosen the following benchmark points at $\tan \beta=15$.

Table 1 lists three benchmark points from [11], of which the BP1 and BP2 belong to the $(1+200)$ model and BP3 to the general non-universal gaugino mass model. The corresponding weak scale SUSY and Higgs spectra are listed in Table 2. We have checked that the rather low $\tilde{\tau}_{1}$ masses are still above the direct and indirect LEP limits [4]. The $M_{3}^{G}$ inputs for the BP1 and BP2 were chosen rather high to ensure that the resulting squark/gluino masses are well above the $8 \mathrm{TeV}$ LHC search limits [35]. This results in a fairly high values of $M_{2}^{G}$, so that the corresponding wino $\left(\chi_{1}^{ \pm}, \chi_{2}^{0}\right)$ masses are $\gtrsim 600 \mathrm{GeV}$. The resulting $\delta a_{\mu}$ is in the range of 1.6-1.9 $\sigma$. For BP3, all the three gaugino mass inputs are independent. Thus we have chosen a large enough $M_{3}^{G}$ to correspond to squark/gluino masses even beyond the reach of $13 \mathrm{TeV}$ LHC along with a modest $M_{2}^{G}$ to correspond to wino $\left(\chi_{1}^{ \pm}, \chi_{2}^{0}\right)$ mass $\approx 460 \mathrm{GeV}$. The resulting $a_{\mu}^{S U S Y}$ is within $0.26 \sigma$ of the experimental value. However, we shall see below that this benchmark point can be easily tested with the available 8 $\mathrm{TeV}$ data

We note from Table 2 that the left slepton $\left(\tilde{l}_{L}\right)$, representing left selectron and smuon is always $\approx 20 \%$ lighter than the charged and neutral wino $\left(\chi_{1}^{ \pm}, \chi_{2}^{0}\right)$. It is a robust feature of these nonuniversal gaugino mass models, following from a small and universal $m_{0}$ - the smallness being required by the bulk annihilation region of dark matter relic density. It ensures that the produced $\chi_{1}^{ \pm}, \chi_{2}^{0}$ pair dominantly decay via the left sleptons, resulting in viable trilepton and same sign dilepton signals with two hard leptons. The latter signal is unaffected even if the left sleptons mass 
becomes very close to the wino mass. These multilepton signals will become unviable only if the left slepton becomes heavier than the wino, which will require a large $m_{0}$. This means one has to sacrifice either the bulk annihilation region of dark matter relic density or the common scalar mass for the left and right sleptons. With these two resonable constraints, the muon $\mathrm{g}-2$ satisfying SUSY models predict trilepton and even more robust same sign dilepton siganls at LHC. Note that for simplicity we have assumed the same low $m_{0}$ value of the slepton sector for the squark sector as well. However, this assumption has no impact on our result. Assuming a large $m_{0}$ for the squark sector instead will only increase the squark masses, which has no effect however on the electroweak SUSY signal of our interest. LHC searches for other muon g-2 satisfying SUSY models have been discussed in [36-38].

\section{Signal and Background}

As discussed in the previous section the NUGM models provide a framework which can accommodate the bulk annihilation region of the right DM relic density as well as the required muon $\mathrm{g}-2$. It implies that the wino masses are in the range of $400-700 \mathrm{GeV}$, which implies pair production of $\tilde{\chi}_{1}^{ \pm} \widetilde{\chi}_{2}^{0}$ at the LHC with a sizable cross section. Once this pair is produced in proton-proton collision, their cascade decays lead to the final state containing hard leptons along with lightest neutralinos $\left(\widetilde{\chi}_{1}^{0}\right)$, which is assumed to be the lightest SUSY particle(LSP). The presence of LSPs in the final state results in an imbalance in the measured transverse momentum $\left(\not p_{T}\right)$ due to its very weak interaction with the detector. Hence the decay channel,

$$
\tilde{\chi}_{1}^{ \pm} \widetilde{\chi}_{2}^{0} \rightarrow\left(\ell^{ \pm} v \widetilde{\chi}_{1}^{0}\right)\left(\ell^{+} \ell^{-} \widetilde{\chi}_{1}^{0}\right)
$$

with $\ell=\mathrm{e}, \mu$, leads to a trilepton signal having same flavor opposite sign(SFOS) leptons or a same sign dilepton(SSDL) signal, each with a reasonable amount of $p_{T}$. It is to be noticed that this signal is hadronically quiet which can be exploited to get rid of standard model(SM) backgrounds. The dominant SM background is due to the WZ production with the leptonic decays of $\mathrm{W}$ and $\mathrm{Z}$ boson providing identical final states like the signal events. In addition, the pair production of top quarks with the semi leptonic decays, $t \rightarrow b W \rightarrow b \ell v$, and semileptonic decay of one of the b-quark leads to three lepton final states. Besides these two dominant SM backgrounds, there are other sources of backgrounds, e.g. from $\mathrm{WW}, \mathrm{WZ}$ and $\mathrm{W} \gamma / \mathrm{Z} \gamma$ production, where the decay hadronic jets from W/Z can fake as leptons. However, these backgrounds are expected to be very small. In the present analysis, we consider only the SM backgrounds due to the top pair and WZ production. It is to be noted that in comparison with the SM backgrounds the leptons and $\not{ }_{T}$ in the signal are expected to be harder, since they originate from comparatively more massive particles like $\tilde{\chi}_{1}^{ \pm}$and $\widetilde{\chi}_{2}^{0}$. We have exploited all these signal characteristics to isolate signal events from large background samples.

The signal and background events are simulated using PYTHIA6 [39]. In cross section calculation we use CTEQ6L1 [40] for parton distribution function setting both factorization and renormalization scales to $\hat{s}$ - the center of mass energy in the partonic frame. In our simulation we adopt the following strategy to select events:

- Lepton selection: As already mentioned, the signal events are expected to contain two hard leptons due to the large mass gap between the left slepton and the LSP. So we apply hard cuts on the first two leptons and a soft cut on the third lepton. Here leptons are arranged in decreasing order of $p_{T}$. For three lepton case with same flavor opposite sign(SFOS),

$$
p_{T}^{\ell_{1,2,3}} \geq 80,50,10 \mathrm{GeV} ;\left|\eta^{\ell_{1,2,3}}\right| \leq 2.5
$$


and for same sign dilepton events(SSDL),

$$
p_{T}^{\ell_{1,2}} \geq 50,50 \mathrm{GeV} ;\left|\eta^{\ell_{1,2}}\right| \leq 2.5 \text {. }
$$

The isolation of lepton is ensured by the total accompanying transverse energy cut $E_{T}^{a c} \leq 20 \%$ of the $p_{T}$ of the corresponding lepton, where $E_{T}^{a c}$ is the scalar sum of transverse energies of jets within a cone of size $\Delta R(l, j) \leq 0.2$ between jet and lepton. These selection of cuts are very useful in suppressing the background events, which will be discussed later.

- Jet selection: Jets are reconstructed using FastJet [41] with jet size parameter $\mathrm{R}=0.5$ and anti $k_{T}$ algorithm [42]. Jets are selected with following thresholds,

$$
p_{T}^{j} \geq 30 \mathrm{GeV} ;\left|\eta^{j}\right| \leq 3.0 \text {. }
$$

As mentioned before, signal events are hadronically quiet at the parton level where as $t \bar{t}$ background events has reasonable hadronic activities. Hence, vetoing out events having at least one jet drastically reduce the $t \bar{t}$ background by enormous amount, which can be observed from Tables 3-6 below.

- In case of SFOS, we require opposite sign and same flavor dilepton invariant mass should not lie within the range $70-110 \mathrm{GeV}$, i.e if $70<m_{l l}<110$, events are rejected. This cut is applied with a goal to suppress background from WZ, where this dilepton invariant mass is expected to have a peak around $M_{Z}$.

- The transverse missing momentum $\left(\not p_{T}\right)$ is calculated adding the momentum of visible particles vectorially and then reverse its sign. and it is required to be $p_{T}>150 \mathrm{GeV}$.

- Another important observable, the transverse mass is found to be very useful to eliminate SM backgrounds without costing signal events too much. The transverse mass is defined to be,

$$
m_{T}\left(\ell, p_{T}^{\prime}\right)=\sqrt{2 p_{T}^{\ell} p_{T}^{\prime}\left(1-\cos \phi\left(p_{T}^{\ell}, p_{T}^{\prime}\right)\right)}
$$

where $\phi\left(p_{T}^{\ell}, p_{T}^{\prime}\right)$ is the azimuthal angle between lepton and missing transverse momentum. In SFOS case, after applying $m_{\ell \ell}$ cut, the remaining third lepton is used to construct $m_{T}\left(\ell_{3}, p_{T}\right)_{T}$. The $m_{T}$ distribution for leptons coming from $\mathrm{W}$ decay either in top pair production or from $\mathrm{WZ}$ channel is expected to show a jacobian peak around $M_{W}$; hence a cut on $m_{T}\left(\ell_{3}, p_{T}\right)>150 \mathrm{GeV}$ effectively suppresses these backgrounds. The main suppression of the WZ background comes of course from the $m_{\ell \ell}$ cut.

- For SSDL case, transverse mass for each lepton, $m_{T}\left(\ell_{1}^{ \pm}, \not p_{T}\right)$ and $m_{T}\left(\ell_{2}^{ \pm}, \not p_{T}\right)$ are constructed and selection cuts are applied separately requiring these to be more than $100 \mathrm{GeV}$. These cuts are very useful in suppressing the $t \bar{t}$ and WZ backgrounds. In this case a transverse mass cut of the dilepton system with the $p_{T}^{\prime}, m_{T}\left(\ell_{1}+\ell_{2}, p_{T}\right)>125 \mathrm{GeV}$, also helps to suppress these backgrounds further.

The signal and backgrounds are simulated for both LHC energies $8 \mathrm{TeV}$ and as well as $13 \mathrm{TeV}$ which is expected to be the Run 2 LHC energy in the next year. For illustration, the signal rates are estimated for the three representative choices of parameter space as shown in Table 1, and the corresponding mass spectra as presented in Table 2.

\section{Results and Discussions}

We present the summary of events in Table 3-6 for both 8 and $13 \mathrm{TeV}$ energies simulating both signal and SM backgrounds $t \bar{t}$ and WZ, adopting the strategy as described in the previous section. 


\begin{tabular}{|c|c|c|c|c|c|c|c||c|c|}
\hline Proc & NoE & $\sigma(\mathrm{fb})$ & $3 \ell$ & $p_{T}^{\prime}$ & $m_{l l} \neq$ & $m_{T}\left(\ell_{3}, p_{T}^{\prime}\right)$ & Jet Veto(JV) & \multicolumn{2}{c|}{$\sigma \varepsilon_{a c}(\mathrm{fb})$} \\
& & & & $\geq 150$ & $M_{Z} \pm 20$ & $\geq 150$ & & No JV & JV \\
\hline BP1 & $50 \mathrm{~K}$ & 1.8 & 9354 & 7393 & 6728 & 5811 & 3407 & 0.20 & 0.12 \\
BP2 & $50 \mathrm{~K}$ & 0.88 & 9591 & 7909 & 7318 & 6409 & 3707 & 0.11 & 0.06 \\
BP3 & $50 \mathrm{~K}$ & 11.3 & 7254 & 4996 & 4263 & 3543 & 2180 & 0.80 & 0.49 \\
\hline$t \bar{t}: 0-200$ & $2 \mathrm{M}$ & 88400 & 689 & 15 & 13 & 1 & 0 & 0.04 & 0.00 \\
$t \bar{t}: 200-500$ & $0.2 \mathrm{M}$ & 9710 & 238 & 22 & 18 & 7 & 0 & 0.33 & 0.00 \\
$t \bar{t}: 500-$-up & $10^{5}$ & 130 & 288 & 128 & 119 & 44 & 0 & 0.05 & 0.0 \\
\hline$W^{ \pm} Z$ & $0.45 \mathrm{M}$ & 13000 & 525 & 32 & 10 & 10 & 6 & 0.28 & 0.17 \\
\hline
\end{tabular}

Table 3: Event summary for trilepton final state with same flavor opposite sign (SFOS) corresponding to signal and SM backgrounds at $8 \mathrm{TeV}$ energy along with production cross section in LO(third column). The last two columns show the normalized cross-section $\left(\sigma \times\right.$ acceptance efficiency $\left.\left(\varepsilon_{a c}\right)\right)$ without and with jet veto(JV). The $t \bar{t}$ is simulated for three $\hat{p}_{T}$ bins.

\begin{tabular}{|c|c|c|c|c|c|c|c|c|c|c|}
\hline \multirow[t]{2}{*}{ Proc } & \multirow[t]{2}{*}{$\mathrm{NoE}$} & \multirow[t]{2}{*}{$\sigma(\mathrm{fb})$} & \multirow{2}{*}{$\begin{array}{l}n_{\ell}=2 \\
\text { SSDL }\end{array}$} & \multirow{2}{*}{$\begin{aligned} & p_{T}^{\prime} \\
\geq & 150\end{aligned}$} & \multirow{2}{*}{$\begin{array}{c}m_{T}\left(\ell_{1}, p_{T}{ }_{T}\right) \\
\geq 100\end{array}$} & \multirow{2}{*}{$\begin{array}{c}m_{T}\left(\ell_{2}, p_{T}^{\prime}\right) \\
\geq 100\end{array}$} & \multirow{2}{*}{$\begin{array}{c}m_{T}\left(\ell_{1}+\ell_{2}, p_{T}^{\prime}\right) \\
\geq 125\end{array}$} & \multirow{2}{*}{$\begin{array}{c}n_{j}=0 \\
\text { JetVeto }(\mathrm{JV})\end{array}$} & \multicolumn{2}{|c|}{$\overline{\sigma \times \varepsilon(\mathrm{fb})}$} \\
\hline & & & & & & & & & No JV & JV \\
\hline BP1 & $50 \mathrm{~K}$ & 1.8 & 9249 & 7180 & 6953 & 6082 & 5921 & 3338 & 0.21 & 0.12 \\
\hline BP2 & $50 \mathrm{~K}$ & 0.88 & 9692 & 7876 & 7642 & 6753 & 6790 & 3678 & 0.12 & 0.06 \\
\hline BP3 & $50 \mathrm{~K}$ & 11.3 & 6541 & 4260 & 4131 & 3548 & 3469 & 2031 & 0.78 & 0.45 \\
\hline$t \bar{t} 0-200$ & $2 \mathrm{M}$ & 88400 & 148 & 3 & 1 & $\overline{0}$ & $\overline{0}$ & $\overline{0}$ & $\overline{0}$ & $\overline{0}$ \\
\hline$t \bar{t} 200-500$ & $0.2 \mathrm{M}$ & 9710 & 75 & 10 & 5 & 0 & 0 & 0 & 0 & 0 \\
\hline$t \bar{t} 500$-up & $0.1 \mathrm{M}$ & 130 & 474 & 203 & 56 & 2 & 1 & 0 & 0.001 & 0 \\
\hline$W^{ \pm} Z$ & $.45 \mathrm{M}$ & 13000 & 568 & 14 & 12 & 3 & 1 & 1 & 0.003 & 0.003 \\
\hline
\end{tabular}

Table 4: Same as Table 3, but for same sign dilepton case(SSDL).

Table 3 presents the number of trilepton events for $8 \mathrm{TeV}$ energy after each set of cuts as shown on top of each columns. The 2 nd and 3rd columns show the number of events(NoE) simulated and leading order(LO) cross sections(in fb) respectively for each process, where as the fourth column presents the number of events having 3 leptons in the final states passing cut, eq. 14. Note that the $t \bar{t}$ events are simulated for three $\hat{p}_{T}$ bins to consider statistics appropriately in different phase space regions. Here $\hat{p}_{T}$ is the transverse momentum of top quark pair in partonic frame. Notice that selection cuts on $\not p_{T}$ and $m_{T}\left(\ell_{3}, p_{T}\right)$ are very effective to suppress background events, in particular $t \bar{t}$ events, where as dilepton invariant mass $\left(m_{\ell \ell}\right)$ cut suppresses mainly WZ background, with little effect on signal events. Eventually the jet veto(JV) criteria, i.e. reject events if there exist jets in the final states, reduces the $t \bar{t}$ backgrounds drastically with a mild effect on signal events. As noted earlier, in signal events presence of jets are mainly due to initial state radiation; and hence the signal events are not expected to have many hard jets unlike the $t \bar{t}$ background. The last two columns display the final cross sections multiplying by acceptance efficiency for both cases, with and without jet veto. Clearly, jet veto completely brings down the top backgrounds to a negligible level, but residual WZ background remains.

In Table 4 we show event summary for SSDL case at $8 \mathrm{TeV}$ energy. In this case we apply same set of selection cuts as discussed before, but in addition two more selection cuts $m_{T}\left(\ell_{1}, \not_{T}^{\prime}\right)$ and $m_{T}\left(\ell_{2}, p_{T}\right)$ are used with a purpose to suppress mainly WZ background. It is motivated by the fact that in $\mathrm{WZ}$ channel two leptons always come from $\mathrm{W}$ and $\mathrm{Z}$ decays, and the one coming from $\mathrm{W}$ decay will not kinematically pass the $m_{T}>100 \mathrm{GeV}$ cut. In contrast the signal events, where leptons originate from heavier $\chi_{1}^{ \pm}$and $\widetilde{\chi}_{2}^{0}$ decays pass the $m_{T}>100 \mathrm{GeV}$ cut for both the 


\begin{tabular}{|c|c|c|c|c|c|c|c||c|c|}
\hline Proc & NoE & $\sigma(\mathrm{fb})$ & $3 \ell$ & $p_{T}^{\prime}$ & $m_{l l} \neq$ & $m_{T}\left(\ell_{3}, p_{T}^{\prime}\right)$ & \multicolumn{2}{|c||}{$\mathrm{n}_{\mathrm{j}}=0$} & \multicolumn{2}{|c|}{$\sigma \times \varepsilon(\mathrm{fb})$} \\
& & & & $>150$ & $m_{Z} \pm 20$ & $>150$ & Jet Veto(JV) & No JV & JV \\
\hline BP1 & $50 \mathrm{~K}$ & 7.4 & 8880 & 7123 & 6459 & 5519 & 2883 & 0.81 & 0.43 \\
BP2 & $50 \mathrm{~K}$ & 4.2 & 9290 & 7753 & 7168 & 6228 & 3084 & 0.52 & 0.26 \\
BP3 & $50 \mathrm{~K}$ & 38 & 6941 & 4882 & 4196 & 3470 & 1885 & 2.64 & 1.42 \\
\hline$t \bar{t} 0-200$ & $30 \mathrm{M}$ & 362000 & 10983 & 543 & 468 & 48 & 1 & 0.57 & 0.012 \\
$t \bar{t} 200-500$ & $4 \mathrm{M}$ & 40000 & 4286 & 615 & 549 & 175 & 6 & 1.75 & .06 \\
$t \bar{t} 500-\mathrm{Inf}$ & $0.1 \mathrm{M}$ & 810 & 300 & 129 & 116 & 46 & 0 & 0.37 & 0 \\
\hline$W^{ \pm} Z$ & $4 \mathrm{M}$ & 26000 & 4330 & 902 & 97 & 84 & 45 & 0.54 & 0.29 \\
\hline
\end{tabular}

Table 5: Event summary for SFOS case, same as Table 3 but for $13 \mathrm{TeV}$ energy.

\begin{tabular}{|c|c|c|c|c|c|c|c|c|c|c|}
\hline \multirow[t]{2}{*}{ Proc } & \multirow[t]{2}{*}{ Evt } & \multirow[t]{2}{*}{$\sigma(\mathrm{fb})$} & \multirow{2}{*}{$\begin{array}{l}n_{\ell}=2 \\
\text { SSDL }\end{array}$} & \multirow{2}{*}{$\begin{aligned} & p_{T}^{\prime} \\
\geq & 150\end{aligned}$} & \multirow{2}{*}{$\begin{array}{c}m_{T}\left(\ell_{1}, p_{T}^{\prime}\right) \\
\geq 100\end{array}$} & \multirow{2}{*}{$\begin{array}{l}m_{T}\left(\ell_{2}, p_{T}^{\prime}\right) \\
\quad \geq 100\end{array}$} & \multirow{2}{*}{$\begin{array}{c}m_{T}\left(\ell_{1}+\ell_{2}, p_{T}^{\prime}\right) \\
\geq 125\end{array}$} & \multirow{2}{*}{$\begin{array}{c}n_{j}=0 \\
\text { JetVeto }(\mathrm{JV})\end{array}$} & \multicolumn{2}{|c|}{$\sigma \times \varepsilon(\mathrm{fb})$} \\
\hline & & & & & & & & & No JV & JV \\
\hline BP1 & $50 \mathrm{~K}$ & 7.4 & 8803 & 6961 & 6721 & 5882 & 5710 & 2925 & 0.84 & 0.44 \\
\hline BP2 & $50 \mathrm{~K}$ & 4.2 & 9663 & 7942 & 7691 & 6772 & 6582 & 3145 & 0.55 & 0.26 \\
\hline BP3 & $50 \mathrm{~K}$ & 38 & 6420 & 4295 & 4131 & 3481 & 3383 & 1821 & 2.57 & 1.38 \\
\hline$t \bar{t} 0-200$ & $30 \mathrm{M}$ & 362000 & 2566 & 165 & 30 & 2 & 1 & 0 & 0.01 & $\overline{0}$ \\
\hline$t \bar{t} 200-500$ & $4 \mathrm{M}$ & 40000 & 1835 & 297 & 129 & 17 & 13 & 0 & 0.13 & 0 \\
\hline$t \bar{t} 500$-up & $0.1 \mathrm{M}$ & 810 & 546 & 255 & 74 & 9 & 5 & 0 & 0.04 & 0 \\
\hline$W^{ \pm} Z$ & $4 \mathrm{M}$ & 26000 & 4607 & 157 & 104 & 2 & 2 & 1 & 0.013 & .006 \\
\hline
\end{tabular}

Table 6: Event summary for SSDL, same as Table 4 but for $13 \mathrm{TeV}$ energy.

leptons. Finally, as indicated by the Table 4, the level of backgrounds cross sections turn out to be negligible. So the discovery limit in the SSDL channels is determined essentially by the signal size.

Similarly we simulate signal and background events for $13 \mathrm{TeV}$ energy using same set of cuts for both SFOS and SSDL cases, which are presented in Tables 5 and 6 respectively. The pattern of suppression of background events with respect to signal events are more or less the same as observed before. However, effect of jet veto kills signal events a little more than at $8 \mathrm{TeV}$ due to the fact that hadronic activities from ISR/FSR are more at this higher energy.

Finally we summarize our results presenting signal and background cross sections along with the signal significance $(S / \sqrt{B})$ in Tables 7 and 8 for SFOS and SSDL cases respectively. The significance are estimated for integrated luminosity $20 \mathrm{fb}^{-1}$ and $100 \mathrm{fb}^{-1}$ corresponding to 8 and $13 \mathrm{TeV}$ LHC energies. In calculating both signal and background cross sections, we have taken into account the next to leading order effect by multiplying the K-factors for each cases. For example, for $t \bar{t}$ and $\mathrm{WZ}$ processes, we multiply cross sections by 1.6 [43] and 1.7 [44], where as for signal it is 1.5 [45]. Although these K-factors are derived for $14 \mathrm{TeV}$ energy, they are not expected to be very different at 8 and $13 \mathrm{TeV}$.

Table 7 shows the summary of the tri-lepton(SFOS) channel results. We see from this Table that the BP3 corresponding to modest wino $\left(\chi_{1}^{ \pm}, \chi_{2}^{0}\right)$ mass of $\approx 460 \mathrm{GeV}$ can be probed at 5(6) $\sigma$ level with 24(15) trilepton signal events without(with) jet veto from the available $20 \mathrm{fb}^{-1}$ data at 8 $\mathrm{TeV}$. Even without a dedicated search with the model, it may be reasonable to assume that trilepton signal of this size could not be missed in generic search of chargino-neutralino pair production events. On the other hand for BP1 and BP2, corresponding to wino mass $m_{\chi_{1}^{ \pm}}, m_{\chi_{2}^{0}} \gtrsim 600$ $\mathrm{GeV}$, one expects only a couple of trilepton signal events at a significance level $<2 \sigma$ with the 


\begin{tabular}{|c|c|c|c|c|}
\hline Process & $\begin{array}{c}8 \mathrm{TeV} \\
\text { No JV }\end{array}$ & $\begin{array}{c}8 \mathrm{TeV} \\
\text { JV }\end{array}$ & $\begin{array}{c}13 \mathrm{TeV} \\
\text { No JV }\end{array}$ & $\begin{array}{c}13 \mathrm{TeV} \\
\text { JV }\end{array}$ \\
\hline$t \bar{t}$ & 0.67 & - & 4.3 & 0.11 \\
$\mathrm{WZ}$ & 0.48 & 0.29 & 0.92 & 0.5 \\
\hline Total Bg & 1.15 & 0.29 & 5.22 & 0.61 \\
\hline BP1 & 0.3 & 0.18 & 1.21 & 0.64 \\
$\frac{S}{\sqrt{B}}$ & 1.25 & 1.5 & 5.3 & 8.2 \\
\hline BP2 & 0.165 & 0.09 & 0.78 & 0.39 \\
$\frac{S}{\sqrt{B}}$ & 0.68 & 0.74 & 3.4 & 5 \\
\hline BP3 & 1.2 & 0.73 & 3.96 & 2.13 \\
$\frac{S}{\sqrt{B}}$ & 5.0 & 6. & 17.3 & 27 \\
\hline
\end{tabular}

Table 7: Total background and signal cross sections(in fb) for trilepton final states after all selection cuts corresponding to each benchmark point. Note that these cross-sections are obtained by multiplying the background and signal crosssections of Tables 3 and 5 by the appropriate K-factors as described in the text. The significance are computed for integrated luminosities $20 \mathrm{fb}^{-1}$ and $100 \mathrm{fb}^{-1}$ for 8 and $13 \mathrm{TeV}$ energies respectively.

\begin{tabular}{|c|c|c|c|c|}
\hline Process & $\begin{array}{c}8 \mathrm{TeV} \\
\text { No JV }\end{array}$ & $\begin{array}{c}8 \mathrm{TeV} \\
\text { JV }\end{array}$ & $\begin{array}{c}13 \mathrm{TeV} \\
\text { No JV }\end{array}$ & $\begin{array}{c}13 \mathrm{TeV} \\
\mathrm{JV}\end{array}$ \\
\hline$t \bar{t}$ & .002 & 0 & 0.28 & 0 \\
$\mathrm{WZ}$ & 0.005 & 0.005 & 0.022 & 0.01 \\
\hline Total Bg & 0.007 & .005 & 0.30 & 0.01 \\
\hline $\mathrm{BP} 1$ & 0.31 & 0.18 & 1.26 & 0.66 \\
$\mathrm{~S}$ & 6 & 3.6 & 126 & 66. \\
\hline $\mathrm{BP} 2$ & 0.18 & 0.09 & 0.82 & 0.39 \\
$\mathrm{~S}$ & 3.6 & 1.8 & 82 & 39 \\
\hline $\mathrm{BP} 3$ & 1.17 & 0.67 & 3.85 & 2.07 \\
$\mathrm{~S}$ & 23 & 13 & 385 & 207 \\
\hline
\end{tabular}

Table 8: Same as Table 7, but for SSDL case. In this case we show the expected number of signal events for the integrated luminosities of $20 \mathrm{fb}^{-1}$ and $100 \mathrm{fb}^{-1}$ for 8 and $13 \mathrm{TeV}$ respectively. The corresponding $S / \sqrt{B} \geq 10$ for all cases. 
available $20 \mathrm{fb}^{-1}$ data at $8 \mathrm{TeV}$. But with the $100 \mathrm{fb}^{-1}$ data at $13 \mathrm{TeV}$ one can probe BP1(BP2) at a significance level of $\sim 8 \sigma(5 \sigma)$ with $60(40)$ trilepton signal events. This means that even with a $20 \mathrm{fb}^{-1}$ data at $13 \mathrm{TeV}$ a negative search result can rule out wino $\left(\chi_{1}^{ \pm}, \chi_{2}^{0}\right)$ masses upto 600-700 $\mathrm{GeV}$ at $>2 \sigma$ level. This will essentially cover the non-universal gaugino mass models satisfying muon g-2 anomaly up to $2 \sigma$ level.

The summary of the corresponding results for the SSDL channel is shown in Table.8. In this case possibility of observing signal events is more promising due to the presence of tiny backgrounds. Indeed the $S / \sqrt{B}$ ratio is $\geq 10$ for all the cases studied here so that the discovery limit is essentially determined by the number of signal events. Therefore, we show this number here instead of the $S / \sqrt{B}$ ratio. We see from this table that the BP3, corresponding to modest wino mass of $\approx 460 \mathrm{GeV}$, can be probed with 23(13) SSDL signal events without(with) jet veto from the available $20 \mathrm{fb}^{-1}$ data at $8 \mathrm{TeV}$. Again it is reasonable to assume that a signal of this size cannot be missed even in a generic search of chargino-neutralino pair production with this data. For BP1 and $\mathrm{BP} 2$, corresponding to wino mass $\gtrsim 600 \mathrm{GeV}$, one expects only 4-6 and 2-3 SSDL signal events respectively. This falls short of a conservative discovery limit of at least 5-6 events. With the 100 $f b^{-1}$ data at $13 \mathrm{TeV}$ one can probe BP1(BP2) at a significance level of $\approx 26-66 \sigma(15-39 \sigma)$ with 125-65(80-40) SSDL signal events. Thus even with a $20 \mathrm{fb}^{-1}$ data at $13 \mathrm{TeV}$ a negative search results can rule out wino $\left(\chi_{1}^{ \pm}, \chi_{2}^{0}\right)$ masses up to $600-700 \mathrm{GeV}$ at $>5 \sigma$ level. Thus one can unambiguously probe the muon $\mathrm{g}-2$ anomaly satisfying non-universal gaugino mass models at the $13 \mathrm{TeV}$ LHC using either the trilepton or SSDL channels. The SSDL channel has the advantage of a very small background. Besides the SSDL channel also has the advantage of being viable even when the left slepton mass comes very close the wino mass, as discussed earlier.

Recently the ATLAS collaboration have published the analysis of their $20 \mathrm{fb}^{-1}$ data at $8 \mathrm{TeV}$ for chargino pair production signal in the unlike sign dilepton channel [46]. For a $80 \mathrm{GeV}$ LSP $\left(\widetilde{\chi}_{1}^{0}\right)$, they show an expected exclusion region up to $m_{\chi_{1}^{ \pm}}=450-550 \mathrm{GeV}$ at the $95 \%$ C.L. $(\simeq 2 \sigma)$, which is similar to our BP3. There is a preliminary CMS result of search for electroweak charginoneutralino pair production in the tri-lepton channel using their $20 \mathrm{fb}^{-1}$ data at $8 \mathrm{TeV}$ [47]. While most of their analysis focuses on a left slepton mass midway between the $\chi_{1}^{0}$ and $\chi_{2}^{0}\left(=\chi_{1}^{ \pm}\right)$masses, there is one figure( Fig.15b in Ref. [47]) showing the $95 \%$ C.L. exclusion regions in $\chi_{1}^{0}$ and $\chi_{2}^{0}$ masses for left slepton mass close to the latter. The edge of their expected 95\% C.L. $(\simeq 2 \sigma)$ exclusion region for $m_{\chi_{1}^{0}}=80 \mathrm{GeV}$ touches $m_{\chi_{2}^{0}}=600 \mathrm{GeV}$ which is close to our BP1. Thus their expected $2 \sigma$ exclusion limit is stronger than our estimated $1.5 \sigma$ exclusion for BP1 in Table 7 . The main reason for this seems to be their use of b-jet veto instead of a general jet veto, so that they can suppress the $t \bar{t}$ background without sacrificing the SUSY signal. Their b-jet veto criteria have been tuned to their $t \bar{t}$ data. Having no access to this data, we had to rely on the general jet veto to suppress the $t \bar{t}$ background. We hope the CMS collaboration will do a dedicated analysis of their $8 \mathrm{TeV}$ data for chargino-neutralino pair production in tri-lepton and SSDL channels in these non-universal gaugino mass models, where the electroweak super particle masses are fairly well constrained by the dark matter relic density and the muon g-2 anomaly.

\section{Summary}

Non-universal gaugino mass models can naturally account for the dark matter relic density via the bulk annihilation process with relatively light bino LSP and right sleptons in the mass rage of $\sim 100 \mathrm{GeV}$, while accommodating the observed Higgs boson mass of $\sim 125 \mathrm{GeV}$ with $\mathrm{TeV}$ 
scale squark/gluino masses. Some of these models can also account for the observed muon g-2 anomaly via SUSY loops with wino and left sleptons in the mass range of 400-700 GeV. We have investigated the prospect of testing these models via electroweak production of charged and neutral wino pairs at the LHC. The left slepton masses in these models are predicted to lie typically $~ 20 \%$ below the wino mass. Thus one expects robust trilepton and same sign dilepton signals of these models arising from the cascade decays of the charged and neutral wino pair via the left sleptons. In particular the SSDL signal holds even when the left slepton mass lies very close to the wino mass. It also has the advantage of a very small standard model background. Our simulation study shows that the available $8 \mathrm{TeV}$ LHC data is adequate to probe the wino mass range of 400-500 GeV in both the trilepton and the SSDL channels. This mass range of wino covers the muon $\mathrm{g}-2$ range within $0-1 \sigma$ of its observed central value. Moreover the probe can be extended to the wino mass range of 600-700 GeV with the $13 \mathrm{TeV}$ LHC data, which covers the muon g-2 range up to $2 \sigma$ of its central value. Thus the non-universal gaugino mass models satisfying the observed dark matter relic density and the muon g-2 anomaly can be unambiguously tested via electroweak production of the charged and neutral wino pair at the forthcoming $13 \mathrm{TeV}$ run of LHC.

\section{Acknowledgement}

The work of DPR was partly supported by the senior scientist fellowship of the Indian National Science Academy. The authors are also thankful to the organisers of the '13th Workshop on High Energy Physics Phenomenology(WHEPP 13)' held at Puri, Odisha, 12-21st December,2013, where this project was started.

\section{References}

[1] G. L. Kane, "Perspectives on supersymmetry,".

[2] M. Drees, R. Godbole, and P. Roy, "Theory and phenomenology of sparticles: An account of four-dimensional $\mathrm{N}=1$ supersymmetry in high energy physics,".

[3] H. Baer and X. Tata, "Weak scale supersymmetry: From superfields to scattering events,".

[4] Particle Data Group Collaboration, J. Beringer et al., "Review of Particle Physics (RPP)," Phys.Rev. D86 (2012) 010001.

[5] ATLAS Collaboration Collaboration, G. Aad et al., "Observation of a new particle in the search for the Standard Model Higgs boson with the ATLAS detector at the LHC," Phys.Lett. B716 (2012) 1-29, arXiv:1207.7214 [hep-ex].

[6] CMS Collaboration Collaboration, S. Chatrchyan et al., "Observation of a new boson at a mass of $125 \mathrm{GeV}$ with the CMS experiment at the LHC," Phys.Lett. B716 (2012) 30-61, arXiv: 1207.7235 [hep-ex].

[7] S. King, J. Roberts, and D. Roy, "Natural dark matter in SUSY GUTs with non-universal gaugino masses," JHEP 0710 (2007) 106, arXiv: 0705.4219 [hep-ph].

[8] J. R. Ellis, K. Enqvist, D. V. Nanopoulos, and K. Tamvakis, "Gaugino Masses and Grand Unification,” Phys.Lett. B155 (1985) 381.

[9] M. Drees, "Phenomenological Consequences of $N=1$ Supergravity Theories With Nonminimal Kinetic Energy Terms for Vector Superfields," Phys.Lett. B158 (1985) 409.

[10] S. Mohanty, S. Rao, and D. Roy, "Predictions of a Natural SUSY Dark Matter Model for Direct and Indirect Detection Experiments," JHEP 1211 (2012) 175, arXiv:1208.0894 [hep-ph]. 
[11] S. Mohanty, S. Rao, and D. Roy, "Reconciling the muon $g-2$ and dark matter relic density with the LHC results in nonuniversal gaugino mass models," JHEP 1309 (2013) 027, arXiv:1303.5830 [hep-ph].

[12] Muon (g-2) Collaboration Collaboration, G. Bennett et al., "An Improved Limit on the Muon Electric Dipole Moment," Phys.Rev. D80 (2009) 052008, arXiv: 0811.1207 [hep-ex].

[13] M. Davier, A. Hoecker, B. Malaescu, and Z. Zhang, "Reevaluation of the Hadronic Contributions to the Muon g-2 and to alpha(MZ)," Eur.Phys.J. C71 (2011) 1515, arXiv:1010.4180 [hep-ph].

[14] T. Moroi, "The Muon anomalous magnetic dipole moment in the minimal supersymmetric standard model," Phys.Rev. D53 (1996) 6565-6575, arXiv: hep-ph/9512396 [hep-ph].

[15] M. Endo, K. Hamaguchi, S. Iwamoto, and T. Yoshinaga, "Muon $g-2$ vs LHC in Supersymmetric Models," JHEP 1401 (2014) 123, arXiv:1303.4256 [hep-ph].

[16] C. Gnendiger, D. Stckinger, and H. Stckinger-Kim, "The electroweak contributions to $(g-2)_{\mu}$ after the Higgs boson mass measurement," Phys.Rev. D88 no. 5, (2013) 053005, arXiv:1306.5546 [hep-ph].

[17] H. Fargnoli, C. Gnendiger, S. Paehr, D. Stckinger, and H. Stckinger-Kim, "Non-decoupling two-loop corrections to $(g-2)_{\mu}$ from fermion/sfermion loops in the MSSM," Phys.Lett. B726 (2013) 717-724, arXiv:1309.0980 [hep-ph].

[18] H. Fargnoli, C. Gnendiger, S. Paehr, D. Stckinger, and H. Stckinger-Kim, "Two-loop corrections to the muon magnetic moment from fermion/sfermion loops in the MSSM: detailed results," JHEP 1402 (2014) 070, arXiv:1311.1775 [hep-ph].

[19] U. Chattopadhyay and P. Nath, "b - tau unification, $\mathrm{g}(\mathrm{mu})$ - 2, the b - gt; $\mathrm{s}+$ gamma constraint and nonuniversalities," Phys.Rev. D65 (2002) 075009, arXiv: hep-ph/0110341 [hep-ph].

[20] G. Anderson, H. Baer, C.-h. Chen, and X. Tata, "The Reach of Fermilab Tevatron upgrades for SU(5) supergravity models with nonuniversal gaugino masses," Phys.Rev. D61 (2000) 095005, arXiv:hep-ph/9903370 [hep-ph].

[21] K. Huitu, Y. Kawamura, T. Kobayashi, and K. Puolamaki, "Phenomenological constraints on SUSY SU(5) GUTs with nonuniversal gaugino masses," Phys.Rev. D61 (2000) 035001, arXiv:hep-ph/9903528 [hep-ph].

[22] U. Chattopadhyay and D. Roy, "Higgsino dark matter in a SUGRA model with nonuniversal gaugino masses," Phys.Rev. D68 (2003) 033010, arXiv: hep-ph/0304108 [hep-ph].

[23] S. King and J. Roberts, "Natural implementation of neutralino dark matter," JHEP 0609 (2006) 036, arXiv:hep-ph/0603095 [hep-ph].

[24] A. Djouadi, J.-L. Kneur, and G. Moultaka, "SuSpect: A Fortran code for the supersymmetric and Higgs particle spectrum in the MSSM," Comput.Phys.Commun. 176 (2007) 426-455, arXiv:hep-ph/0211331 [hep-ph].

[25] A. Arbey, M. Battaglia, A. Djouadi, and F. Mahmoudi, "The Higgs sector of the phenomenological MSSM in the light of the Higgs boson discovery," JHEP 1209 (2012) 107, arXiv:1207.1348 [hep-ph].

[26] S. Heinemeyer, O. Stal, and G. Weiglein, "Interpreting the LHC Higgs Search Results in the MSSM," Phys.Lett. B710 (2012) 201-206, arXiv:1112.3026 [hep-ph].

[27] B. Allanach, A. Djouadi, J. Kneur, W. Porod, and P. Slavich, "Precise determination of the neutral Higgs boson masses in the MSSM,"JHEP 0409 (2004) 044, arXiv : hep-ph/0406166 [hep-ph].

[28] G. Degrassi, S. Heinemeyer, W. Hollik, P. Slavich, and G. Weiglein, "Towards high precision predictions for the MSSM Higgs sector,” Eur.Phys.J. C28 (2003) 133-143, arXiv: hep-ph/0212020 [hep-ph].

[29] R. Harlander, P. Kant, L. Mihaila, and M. Steinhauser, "Higgs boson mass in supersymmetry to three loops," Phys.Rev.Lett. 100 (2008) 191602, arXiv:0803.0672 [hep-ph]. 
[30] S. P. Martin, "Three-loop corrections to the lightest Higgs scalar boson mass in supersymmetry," Phys.Rev. D75 (2007) 055005, arXiv: hep-ph/0701051 [hep-ph].

[31] G. Belanger, F. Boudjema, P. Brun, A. Pukhov, S. Rosier-Lees, et al., "Indirect search for dark matter with micrOMEGAs2.4," Comput.Phys.Commun. 182 (2011) 842-856, arXiv:1004.1092 [hep-ph].

[32] G. Belanger, F. Boudjema, A. Pukhov, and A. Semenov, "micrOMEGAs: Version 1.3," Comput.Phys.Commun. 174 (2006) 577-604, arXiv : hep-ph/0405253 [hep-ph].

[33] G. Belanger, F. Boudjema, A. Pukhov, and A. Semenov, "MicrOMEGAs: A Program for calculating the relic density in the MSSM," Comput.Phys.Commun. 149 (2002) 103-120, arXiv:hep-ph/0112278 [hep-ph].

[34] E. Komatsu, J. Dunkley, M. R. Nolta, C. L. Bennett, B. Gold, G. Hinshaw, N. Jarosik, D. Larson, M. Limon, L. Page, D. N. Spergel, M. Halpern, R. S. Hill, A. Kogut, S. S. Meyer, G. S. Tucker, J. L. Weiland, E. Wollack, and E. L. Wright, "Five-Year Wilkinson Microwave Anisotropy Probe Observations: Cosmological Interpretation," apjs 180 (Feb., 2009) 330-376, arXiv:0803.0547.

[35] S. Sekmen, "Inclusive SUSY searches at the LHC," arXiv: 1405.4730 [hep-ex].

[36] BayesFITS Group Collaboration, A. Fowlie, K. Kowalska, L. Roszkowski, E. M. Sessolo, and Y.-L. S. Tsai, "Dark matter and collider signatures of the MSSM," Phys.Rev. D88 no. 5, (2013) 055012, arXiv:1306.1567 [hep-ph].

[37] A. Freitas, J. Lykken, S. Kell, and S. Westhoff, "Testing the Muon g-2 Anomaly at the LHC," arXiv:1402.7065 [hep-ph].

[38] M. Chakraborti, U. Chattopadhyay, A. Choudhury, A. Datta, and S. Poddar, "The Electroweak Sector of the pMSSM in the Light of LHC - 8 TeV and Other Data," arXiv: 1404. 4841 [hep-ph].

[39] T. Sjostrand, S. Mrenna, and P. Z. Skands, "PYTHIA 6.4 Physics and Manual,” JHEP 0605 (2006) 026, arXiv:hep-ph/0603175 [hep-ph].

[40] J. Pumplin, D. Stump, J. Huston, H. Lai, P. M. Nadolsky, et al., "New generation of parton distributions with uncertainties from global QCD analysis," JHEP 0207 (2002) 012, arXiv: hep-ph/0201195 [hep-ph].

[41] M. Cacciari, G. P. Salam, and G. Soyez, “FastJet User Manual,” Eur.Phys.J. C72 (2012) 1896, arXiv:1111.6097 [hep-ph].

[42] M. Cacciari, G. P. Salam, and G. Soyez, “The Anti-k(t) jet clustering algorithm,” JHEP 0804 (2008) 063, arXiv:0802.1189 [hep-ph].

[43] N. Kidonakis, "Top Quark Theoretical Cross Sections and pT and Rapidity Distributions," arXiv:1109.3231 [hep-ph].

[44] J. M. Campbell, R. K. Ellis, and C. Williams, "Vector boson pair production at the LHC," JHEP 1107 (2011) 018, arXiv:1105.0020 [hep-ph].

[45] W. Beenakker, M. Klasen, M. Kramer, T. Plehn, M. Spira, et al., "The Production of charginos / neutralinos and sleptons at hadron colliders," Phys.Rev.Lett. 83 (1999) 3780-3783, arXiv:hep-ph/9906298 [hep-ph].

[46] ATLAS Collaboration Collaboration, G. Aad et al., "Search for direct production of charginos, neutralinos and sleptons in final states with two leptons and missing transverse momentum in pp collisions at $\sqrt{s}=8 \mathrm{TeV}$ with the ATLAS detector," arXiv:1403.5294 [hep-ex].

[47] CMS Collaboration Collaboration, V. Khachatryan et al., "Searches for electroweak production of charginos, neutralinos, and sleptons decaying to leptons and W, Z, and Higgs bosons in pp collisions at $8 \mathrm{TeV}$," arXiv:1405.7570 [hep-ex]. 